\title{
SEISMIC INVERSION: ONE STEP OR MANY?
}

\author{
Wendell Wiggins \\ Western Geophysical \\ PO Box 2469 \\ Houston TX 77252 \\ USA
}

Elegant formulations of the seismic inversion method have been developed that map the data directly to the underlying physical quantities (a geologic model) in a single step. Each of these methods either find a geologic model from which synthetic data can be generated to match the observed data (forward modeling) or it numerically reverses the wave propagation to reconstruct the geologic model (direct inversion). While these global inversion methods are appealing from a mathematical point of view, in practice such solutions are almost certainly inadequate to deal with the common problems that plague real seismic data. I will review the problems encountered in deriving a geologic model from seismic data and outline how these problems are currently resolved.

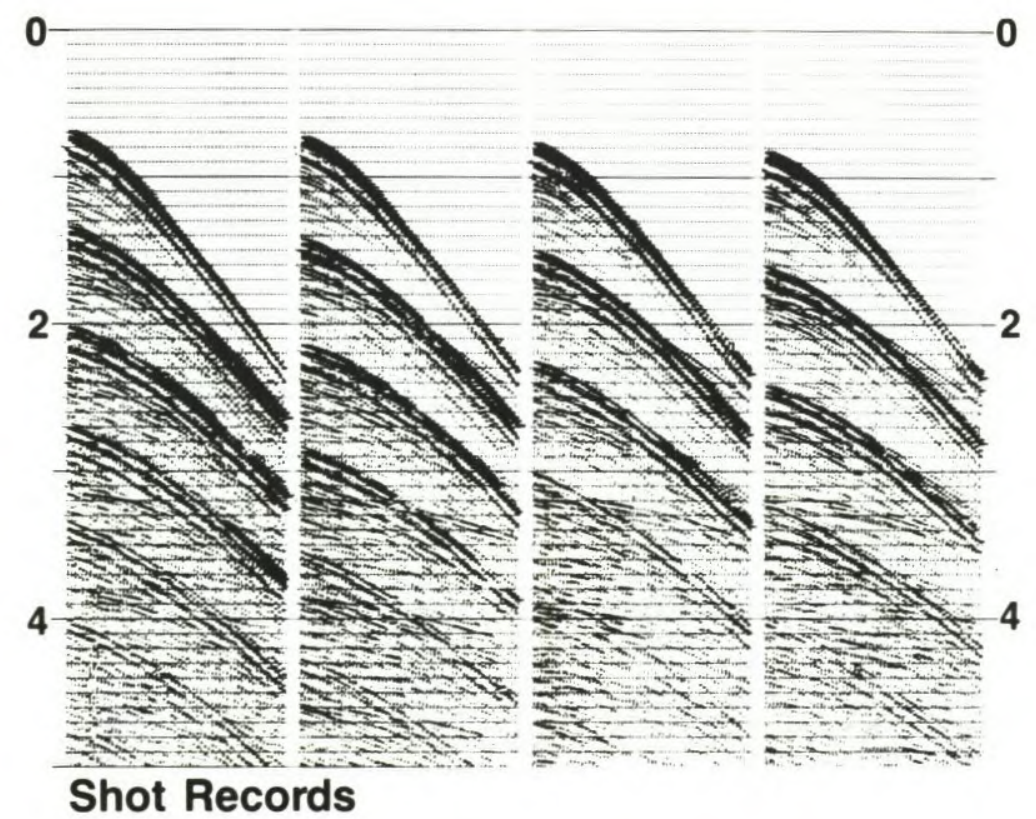

Figure 1 Shot records from a marine survey over a very hard water bottom. Almost no primary reflections are visible except for an event at $3.2 \mathrm{sec}$. The multiples must be attenuated by about $30 \mathrm{~dB}$ before primary reflections are clearly visible before stack. 


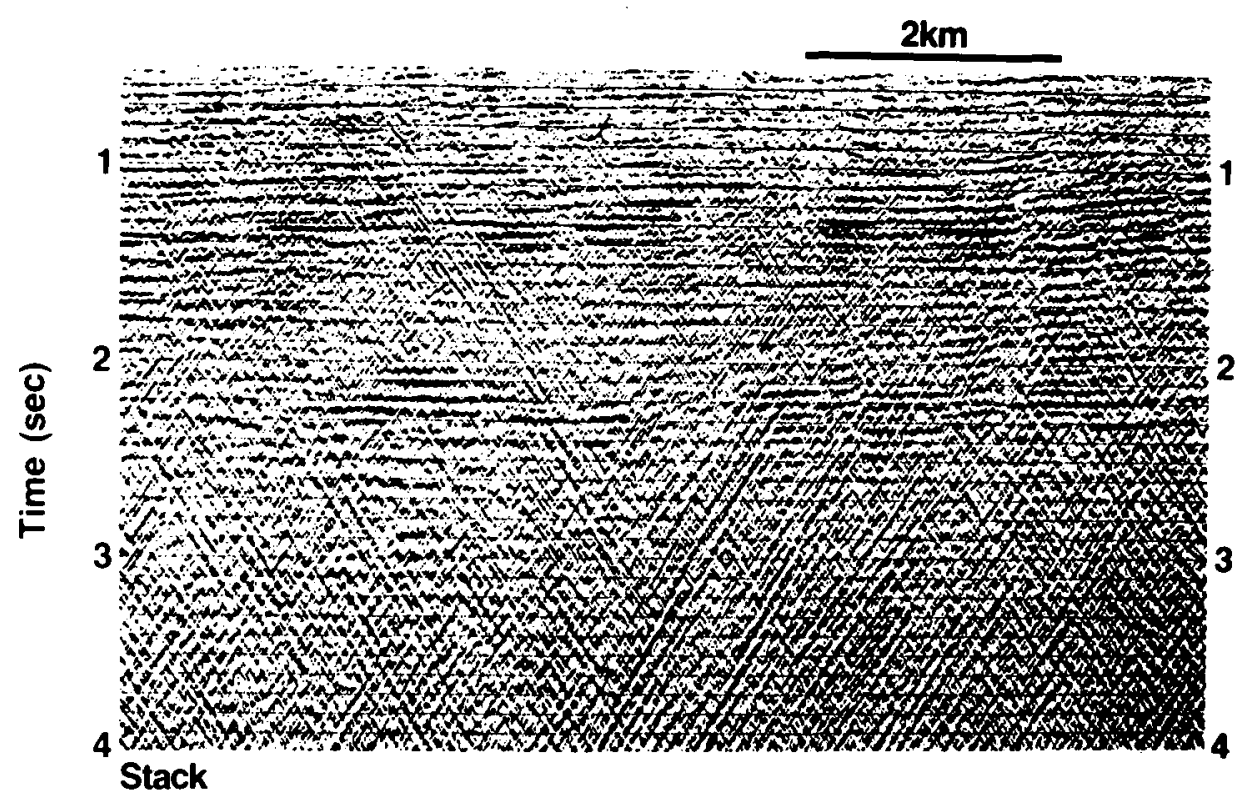

Figure 2 A stacked section from a marine survey. The steeply dipping signal seen uniformly across the section comes from out-of-plane scattering just below the water bottom.

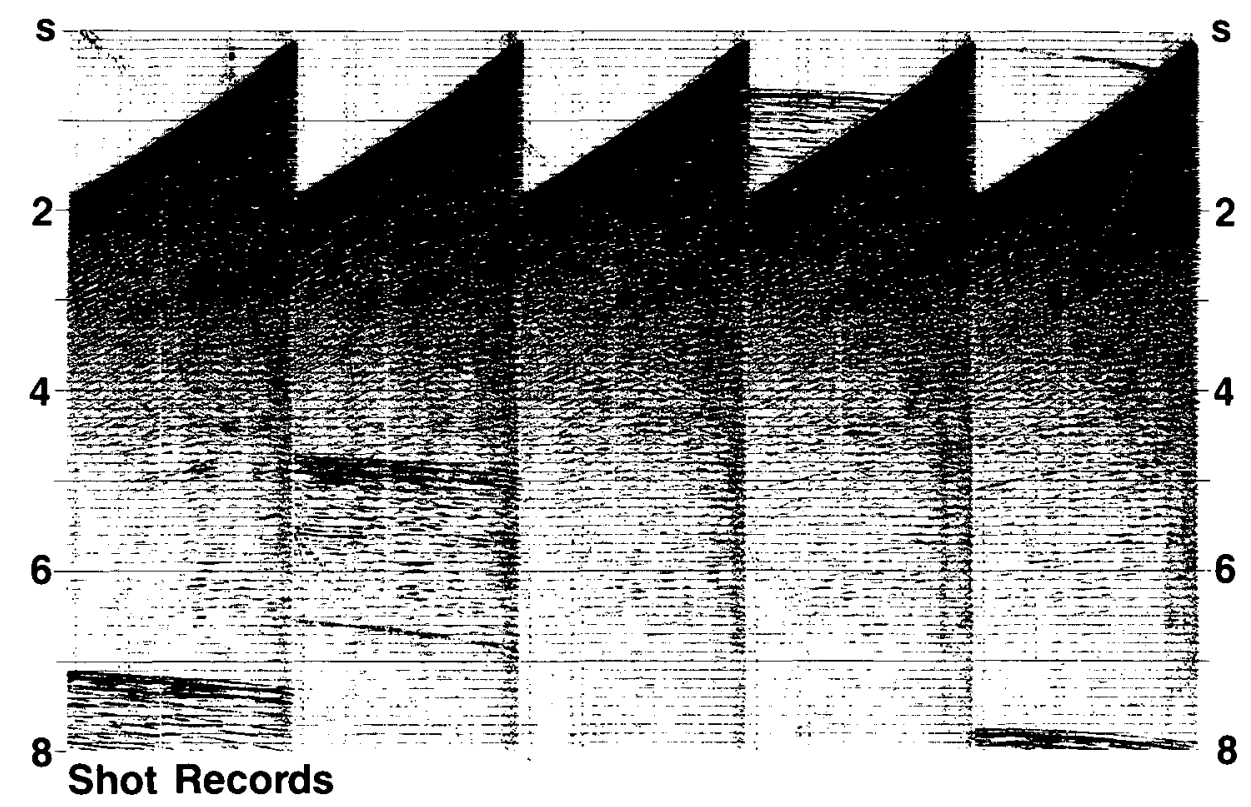

Figure 3 Data recorded while another seismic survey was being shot nearby. The strong irregularly timed event is the direct arrival from the other survey's air guns. 
Noise of many types may contaminate the data. Mode conversions, multiple scattering, refractions and other kinds of wave propagation phenomena, all considered noise in conventional processing, might be accounted for by a very sophisticated, perhaps elastic-wave inversion scheme.

For example, the strong water bottom multiples shown in figure 1 could be dealt with in a global inversion if both transmission and reflection at the water bottom are included in the reverse wave propagation. In fact, these multiples have been most successfully treated in field data only by explicit attenuation methods that discriminate against them on the basis of moveout velocity or model them by wave extrapolation through the water layer and subsequently subtract the modeled multiples.

Figure 2 shows a marine survey contaminated by scattering from hard spots in the strata just below the water bottom. These signals can be considered as part of the geology to be recovered by a global inversion scheme but since they lie out of the plane of the 2-D survey, they can be dealt with only by a full $3-D$ inversion. The essentially 3-D distribution of noise generators and the typically 3-D nature of geologic structures requires that inversion schemes that seek to go beyond current practice be fully 3-dimensional.

Other interfering sources including air waves, nearby seismic crews in marine surveying or power-line noise on land cannot be inverted since they are not part of the desired geologic model nor are they necessary predictable. Figure 3 shows a shot record from a survey where another seismic crew was simultaneously shooting nearby. The interfering direct arrival is quite strong compared to the desired reflections arriving at the same time. The difference in moveout between the desired and interfering events will often attenuate the interference when the data is stacked. A more effective discrimination is obtained by using amplitude dependent scaling of the data (Lynn et al., 1987).

Figure 4 shows a record contaminated by electrical power noise. Although inversion methods designed to handle reflected seismic waves would not recognize this monochromatic noise as objectionable, adaptive filtering designed to rec-

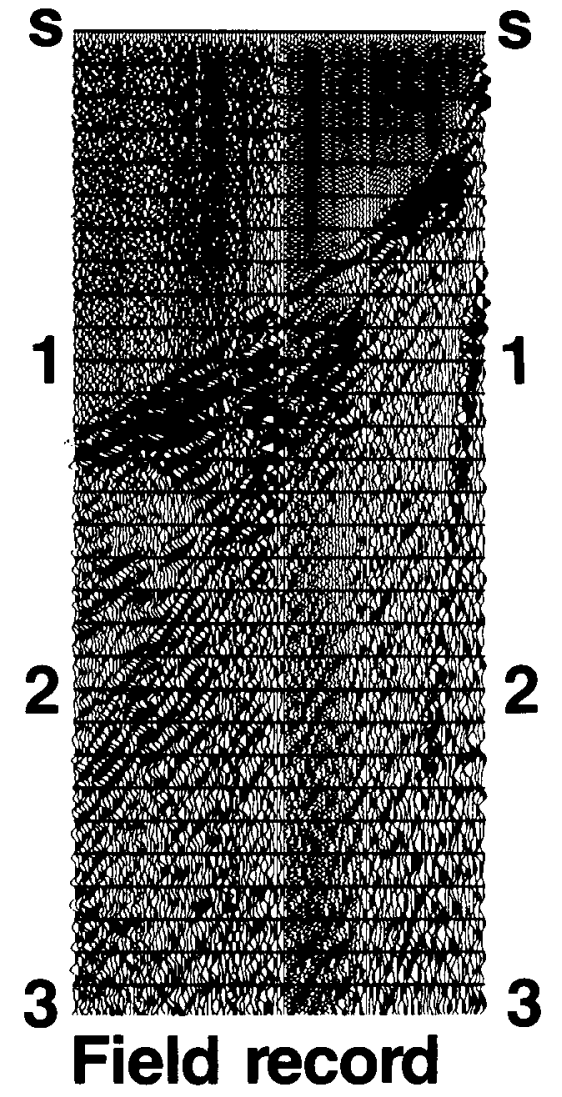

Figure $4 \mathrm{~A}$ shot record containing strong power line interference.

ognize the nearly stationary phase of the interference will nearly perfectly remove it.

Neither missing shots, bad recording channels, nor cable feathering will be easily accommodated by an inversion scheme that expects to find regularly sampled data. Figure 5 shows the layout of a land seismic survey. Surveys on land are typically irregular due to natural and cultural obstacles and the demands of economy. In marine surveys, the recording cable is pushed sideways in an irregular and unpredictable manner to likewise produce irregular survey sampling. Inversion schemes must consequently be designed to deal with irregularly sampled data and arrive at an estimate of the geology that is not unnecessar- 
ily affected by the survey geometry.

Thus, for a wide variety of practical reasons, seismic inversion should not be implemented in a single step. Inversion implemented by many successive steps allows one to modify the individual parts to account for noise and other problems. Examination of the data after each significant step will determine whether that steps goals have been accomplished and whether the data is ready for the next step.

Structural inversion aims to reconstruct a spatial map of reflectivity. Therefore, it is essential that each component of a multistep process preserve all the important characteristics of the data. If the goal is simply an angle-averaged reflectivity, each step must preserve amplitude versus time (depth) and dip. If the inversion aims for normal incidence reflectivity or reflectivity versus reflection angle, all filtering and imaging steps preceding the analysis must preserve the signal amplitude as a function of source to receiver offset. Some conventional forms of noise attenuation, DMO, and prestack migration are not necessary faithful in these measures, especially amplitudeversus-offset behavior. The specific function of each process must be analyzed as a part of a mul- tistep inversion and implemented to ensure the fidelity of the data passed on to the next step.

A space- and frequency- domain inversion that is practically equivalent to prestack Kirchhoff migration has been derived by Sullivan and Cohen (1987). In fact, inversion and migration can be completely equivalent if each is viewed as converting properly preconditioned data to a reflectivity map. Beasley and Mobley (1988) have found proper amplitude and phase factors for a space- and time-domain dip moveout (DMO) that preserves amplitude as a function of offset, time and dip. If this DMO is followed by a trueamplitude zero-offset migration, then the combination of DMO and migration is again equivalent to prestack reflectivity inversion.

Practical inversion at the current state of the processing art typically consist of: compensation for source and recording imperfections; suppression of apparently random noise from multiple scattering, from near surface noises, and from identifiable multiple reflections; compensation for attenuation in transmission; correction for missing data by numerical interpolation; imaging by some combination of DMO, stacking, and zero-offset migration or by prestack migration, This multi-

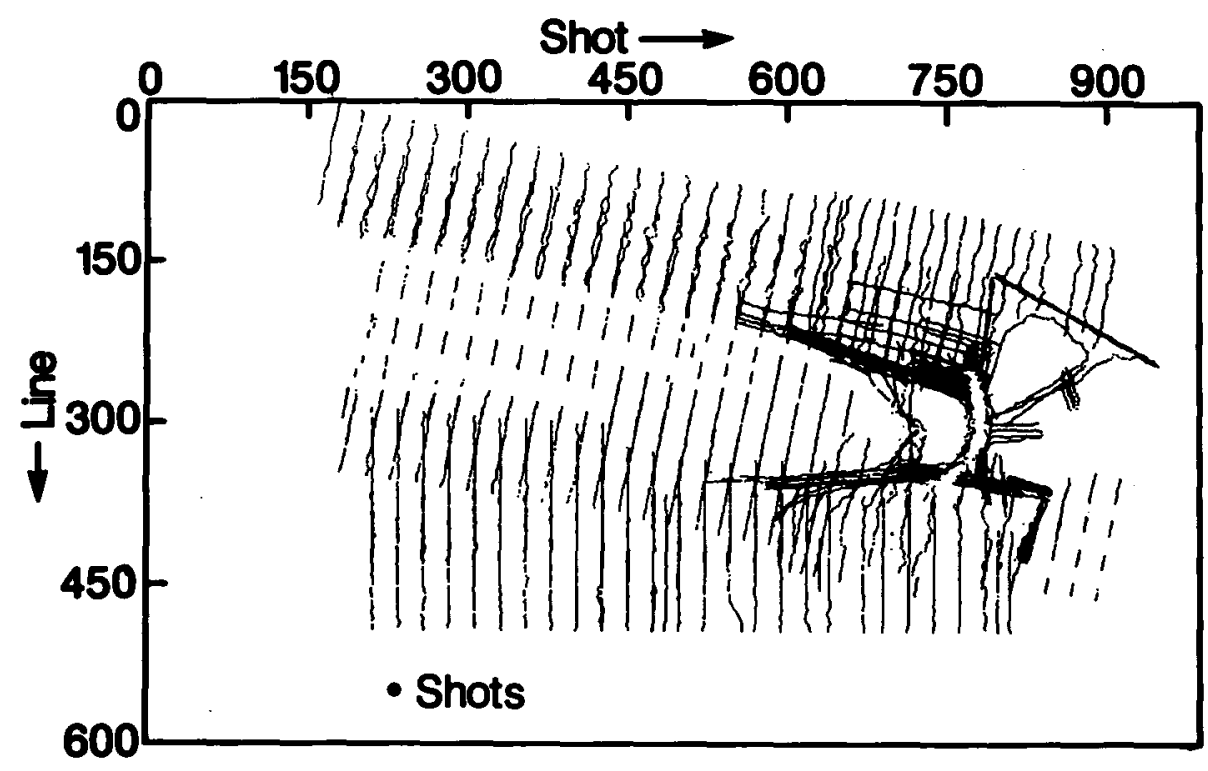

Figure 5 An actual 3-D land survey shot map. Very few land surveys are acquired on a regular, gapless grid. 


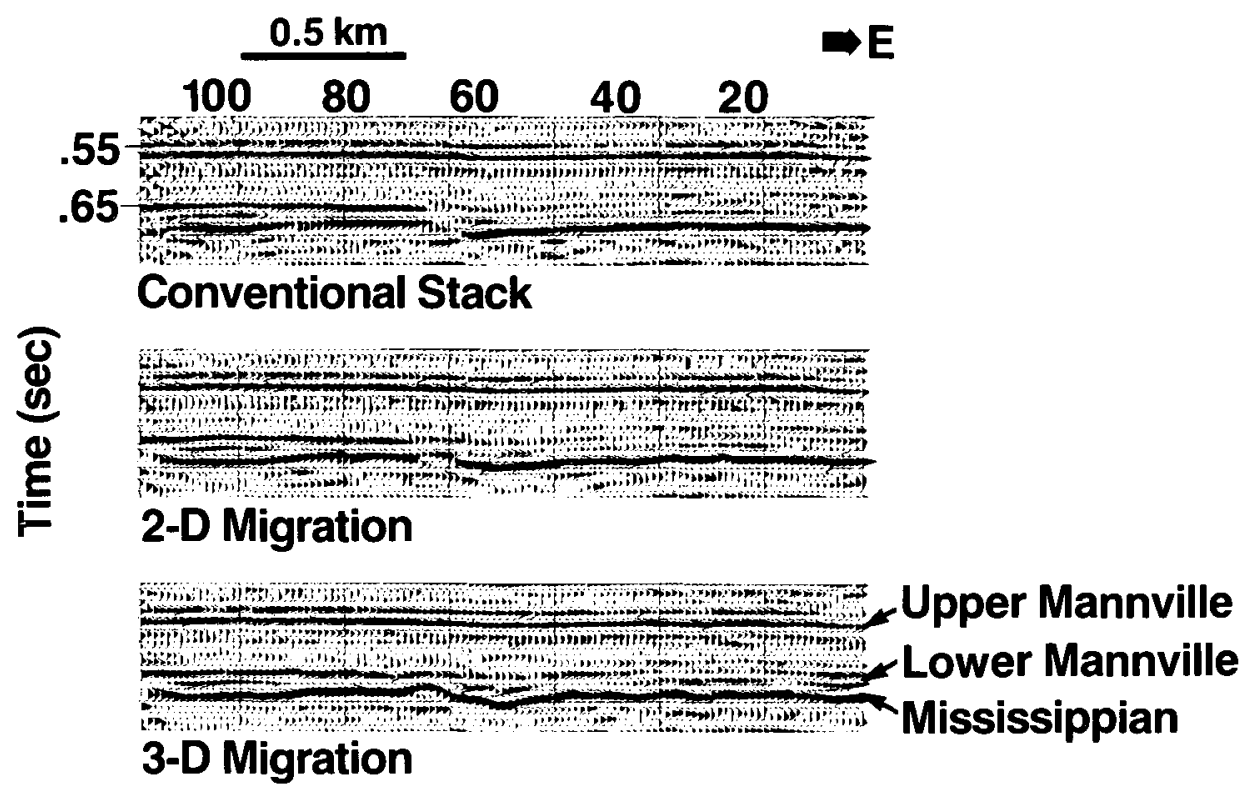

Figure 6 A vertical slice from a 3-D survey. The break in the otherwise flat reflector at $0.65 \mathrm{sec}$ is due to a channel that cuts through the section.

step approach appears likely to remain the best choice for quite some time.

Finally, I emphasize that inversion is almost always a 3-dimensional problem. Even very flat, simple geology may contain spatially limited targets that are expressed over an area of the recorded data. Figure 6 shows a common midpoint stack, the result of migrating that stack in 2-D and the result of 3-D migration. Although the reflector at $0.55 \mathrm{sec}$ is quite flat and featureless, the reflector at $0.65 \mathrm{sec}$ is cut by a channel. Only 3-D migration shows the true shape of this channel. Therefore, in this relatively simple example, the use of 3-D methods is essential. Given the extreme computational expense of some inversion methods in $2-D$, we might prefer a simple $3-D$ inversion to a more precise $2-D$ method.
References

Beasley, C. J. and Mobley, E., 1988, Amplitude and antialiasing treatment in $(x-t)$ domain DMO: 57th Ann. Intl. Mtg. SEG, Expanded Abstracts, 1113-1116.

Lynn, W., Doyle, M. Larner, K. and Marschall, R., 1987, Experimental investigation of interference from other seismic crews: Geophysics 52, 1501-1524.

Sullivan, M.F. and Cohen, J.K., 1987, Prestack Kirchhoff inversion of common-offset data: Geophysics, 52, 745-754. 
